\title{
Research of Tourism Information based on Cloud Computing Platform
}

\author{
Yuting Zhang ${ }^{1}$, Lu Cao ${ }^{2}$, Rui Wang ${ }^{3}$, Yang Zhang ${ }^{4}$, Ying Zhang ${ }^{5}$, Junli \\ Wang ${ }^{6}$, Chen $\mathrm{Hao}^{7}$, Jie Cao ${ }^{8}$, Jiangmin $\operatorname{Tian}^{9}$ \\ ${ }^{1 \sim 9}$ Hainan University, Haikou, Hainan, 570228
}

KEYWORDS: Cloud Computing; Tourism Information; Society Development

\begin{abstract}
With the advent of continuous development of society and the era of mass tourism, tourism is increasingly becoming a major lifestyle of modern people and economic activity in the community, a large tourist information and improving the quality of people's travel, not the traditional travel agency can meet. The traditional travel patterns exist serious information silos, data cannot be integrated, interoperable, applications cannot be interconnected, and the utilization of system resources is not high range of issues. Practice has proved an effective way to solve the drawbacks of the tourism industry is to increase the level of tourism information. In recent years, with the "smart city" proposed "smart tourism," the word is no longer unfamiliar. As a populous country, China's tourism information is also accelerating the construction in progress, on the basis of the existing development and construction based on the "cloud computing" intelligent travel information system, build a "smart tourism cloud" to achieve tourism marketing, management, services in one, visitors can make use of the mobile Internet terminal equipment, active perceivable travel information.
\end{abstract}

\section{Introduction}

Cloud computing is a new development concept, there is no international organization for the cloud given standard definition of different areas from different angles gives the definition of the different descriptive: Wikipedia that" cloud computing "a method is provided as a service to the user's computing model capable of dynamically scalable virtualized resources via the Internet, users do not need to know what kind of infrastructure is used by the international organization for standardization/international electro-technical Commission joint technical Committee (ISO/IECJTCI) from two perspectives define cloud computing: from the "cloud" provider's perspective, "cloud computing" the IT infrastructure and resources as a service to on-demand, according to the amount of the charge provided to the user ; from the "cloud computing" the user's point of view, the user can anytime and anywhere access cloud data and various applications via any device.

"Cloud computing" is a business model, is an Internet-based computing model is super, its computing resources (including interactive capabilities, storage capacity, computing power, etc.) is scalable, dynamic, is virtualized, provided through the service. "Cloud computing" use Pay per use model, which implements network access, the pool of configurable computing resources (eg, networks, servers, storage, applications, and services, etc.) up, convenience and on-demand nature, requires only a minimal amount of management effort or service provider will be able to communicate quickly obtain and release resources. This new computing model is conducive to the 
rational allocation of computing resources, promote energy conservation and improve their utilization. "Cloud computing" will be distributed computing tasks on the computer resource pool configuration, and then obtain the storage space according to their needs, computing power, and a variety of software services. It is a dynamic, easily scalable, and often virtualized computing method implemented via the Internet.

\section{Characteristics of Cloud Computing}

"Cloud computing" has the following characteristics: (1) On-demand self-service consumer can unilaterally acquire computing power on demand, such as server time and network storage, without the need for human interaction with each server provider. (2) Ubiquitous network access exist in the network and accessed through standard mechanisms that promote the function in a heterogeneous "thin client" or "fat client" side platform (such as mobile phones, notebook and PDA) usage of. Terminal devices require the user to use cloud computing technology is very low, does not limit the access area can be at any location, using a variety of terminal devices to obtain application services. (3) Resource pool of computing resources providers on the position-independent resource pool, using a multi-tenant model to provide services to all consumers, according to consumer demand for different physical and virtual resources dynamically allocated or re-allocated. Clients generally do not know the specific location of the resources allocated to, and unable to control the allocation of resources. These resources include storage, processing power, memory, network bandwidth, and virtual machines. (4) To provide fast and flexible capabilities to quickly and flexibly implement extensions, and can be quickly release resources to achieve contraction. For consumers, the resources are available for hire seems to be unlimited, and can be any number of purchasing at any time. (5) By using the premium features are charged according to the amount calculated using the paid service or use pricing model based on advertising to improve resource utilization. For example, the measurement storage bandwidth and computing resources as well as a monthly fee based on the number of user activity.

\section{Tours cloud-related technology architecture}

"Cloud computing" service model from low to high are: Iaas (the infrastructure as a service), Paas (Platform as a Service will), Saas (software as a service).

Iaas-Infrastructure as a service, Infrastructure as a service is actually the data center as a service, or the ability to remotely access computing resources. In essence, rent a physical server, do whatever you want on top of things, and it is part of a data center or data center. Access to very expensive data center resources through a relatively low-cost lease agreement so as to enterprises save money. Applications Mainly animation producers, data mining providers, weather bureau and so on. They write your own programs, responsible for their own operations and analysis, mainly through capacity-parallel cloud computing.

Paas-Platform as a Service Platform as a Service platform hosted remotely through a complete platform for delivery to subscribers, including application development, interface development, database development, storage and testing capabilities. Is a need to download or install, you can send mode operating system and related services through the Internet, which is the development environment as a service to provide.

Saas-software as a service. Internet software as a service is a mode of delivering software through. In short, the software vendor will be deployed on its own server the client software is 
required to hire the service to vendors over the Internet. In this case, the lower the cost of the customer uses the software and even completely without IT maintenance personnel.

\section{Tours Cloud Platform Environment to Build}

In order to make the system intelligent tourism center can be built on a common computing device layer, for computing devices and smart travel subsystem applications were stratified to isolate a common layer of the computing device. Separated the computing device layer also took advantage of the current "cloud computing" services provided by the service provider, so that the various applications of intelligent Tourism System Center has become "cloud computing" is an application service that can put this based on the "cloud computing" intelligent tourism system builders call "intelligent tourism cloud" construct. Based on the "cloud computing" intelligent tourism system "cloud" Construction is the key, "cloud" requires significant processing power and fast requests for capacity for the realization of the "cloud" - "channel" - "terminal" basis.

"Cloud" is equivalent to a data center via the parallel computing and mass storage technology, in the "cloud", so a lot of resources to build vital storage "cloud" data centers. Google is similar to a large virtual machine Google App Engine platform, the traditional infrastructure placed in the platform, and configure the operating environment, developers only need to upload your application package to the platform to a significant reduction in development costs. Based on the "cloud computing" intelligent tourism system in the platform development, system access interface and interact through GAE Big-table database provided for storage and access files through the Google file system GFS.

With only the "cloud" and "terminal" is not enough, we need to graft a bridge between, like a channel connected to the "cloud" and "end", and the broadband network is the important bridge. Telecom operators as "cloud computing" is the largest provider of technology continues to improve, improve the intelligence level of the channel, and also control the "cloud" and "terminal", and strive to provide users with one-stop service.

Terminal refers to the user to accept the "cloud" of the terminal device, not a personal PC, any device access to the Internet, it can be a laptop, desktop, mobile phone or other possible information exchange equipment, including desktop, mobile end and the Web end. For tourists, there are more people through a smart phone or PDA device access, terminal equipment as technology constantly updated via GPS positioning, the user terminal equipment and enjoy not only the service providers but also service, for the user's request is sent to the database, the database response and stored. Making tourism becomes more integrated into life, to enjoy the joy of travel life anytime, anywhere.

\section{Achieve Cloud Function}

Since Google App Engine platform support Java development, and you can use standard Java technology to develop their own applications, java in the development of Web applications have a unique advantage, so the program uses java language development. Development tools MyEclipse7.5, because Google App Engine only supports java 5 and java6, so the platform and development environment for Jdkl.6 Appengine-java-sdk-1.6. Eclipse is an open source, Java-based extensible development platform. On its own, it is only a framework and a set of services for building development environments by plug-in component. However, most users are happy to use Eclipse as a Java IDE, but Eclipse's goal is not only limited to this. Eclipse also includes a plug-in development environment (Plug-in Development Environment, PDE), the main component for the desired expansion of the Eclipse software developers, because it allows them to build seamless 
integration with the Eclipse environment tools. Because Eclipse of everything is plug-in, providing for a Eclipse plug-ins, as well as to provide users with a consistent and unified integrated development environment, developers have all the tools to play the same place.

Attractions in collecting statistical information, a problem that must be faced is: You must take resort information into the memory, and often consume a very long time, the use of Map-Reduce, such work is done only once, when the information is loaded into memory resort after all the work will be performed in memory, statistical information to enhance the speed of the speed will be. When choosing a resort information preload timing is the first time statistical information, which is comparable to delay loading, and only when it is used to initialize. Statistical information is part of the scenic realization of the code is far simpler than imagined. In the process, to have a new scenic spots in each region a noun, it is more object-oriented approach, making it more convenient and

simple. Statistics resort is divided into two parts and there are attractions for area attractions in the region counts and counting.

\section{Conclusion}

Application of cloud computing has become a hot topic in the industry wisdom. Therefore, increasing development and growth of Chinese tourism, we must cater to cater to the trend of the times, closely integrated with the cloud, and ultimately built a sound tourism cloud platform.

\section{REFERENCE:}

[1] Gosinski, Andrzei, Broek, Michael. A tenant-based resource allocation model for scaling Software-as-a-Service applications over cloud computing [J]. Future Generation Computer Systems, 2012,29 (7): 273-286.

[2] Subashini S, Kavitha V. A survey on security issues in service delivery model soft cloud computing [J]. Journal of Network and Computer Applications, 2011,34 (1): 1-11.

[3] Wei Yi, Blake, Brian Service oriented computing and cloud computing. Challenges and opportunities [J] .IEEE Internet Computing, 2010,14 (6): 72-75.

[4] Haggerty.J, Gresty.D, Hegarty.R. Digital in cloud computing systems [J] .Computer Law and Security Report, 2010,26 (3): 304-308. 